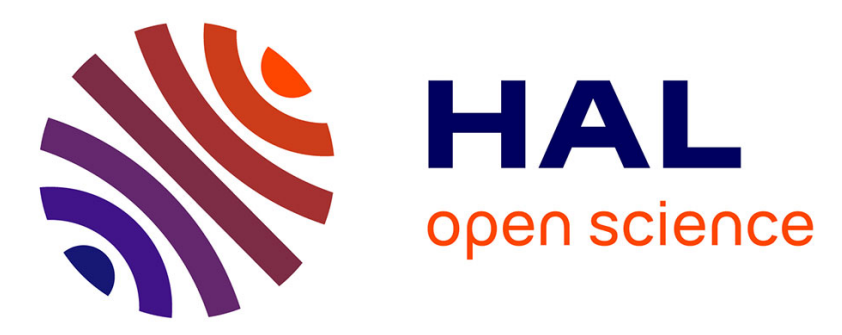

\title{
A QoS-control Framework for Medical Multimedia Data Transmission in CRN Environment
}

\author{
Dramane Ouattara, Mohamed Aymen Chalouf, Omessaad Hamdi, Francine
}

Krief

\section{- To cite this version:}

Dramane Ouattara, Mohamed Aymen Chalouf, Omessaad Hamdi, Francine Krief. A QoS-control Framework for Medical Multimedia Data Transmission in CRN Environment. The nineteenth IEEE Symposium on Computers and Communications ISCC 2014, Jun 2014, Madeira, Portugal. 7 p. hal00998849

\section{HAL Id: hal-00998849 \\ https://hal.science/hal-00998849}

Submitted on 2 Jun 2014

HAL is a multi-disciplinary open access archive for the deposit and dissemination of scientific research documents, whether they are published or not. The documents may come from teaching and research institutions in France or abroad, or from public or private research centers.
L'archive ouverte pluridisciplinaire HAL, est destinée au dépôt et à la diffusion de documents scientifiques de niveau recherche, publiés ou non, émanant des établissements d'enseignement et de recherche français ou étrangers, des laboratoires publics ou privés. 


\title{
A QoS-control Framework for Medical Multimedia Data Transmission in CRN Environment
}

\author{
Dramane Ouattara*, Mohamed Aymen Chalouf ${ }^{\dagger}$, Omessaad Hamdi* and Francine Krief ${ }^{* \ddagger}$ \\ * Univ. Bordeaux, LaBRI, Talence, France. \\ $\ddagger$ IPB, LaBRI, Talence, France. \\ Email: dramane.ouattara@labri.fr, ohamdi@labri.fr, francine.krief@labri.fr. \\ †Univ. Rennes 1, IRISA, Lannion, France. Email: mohamed-aymen.chalouf@irisa.fr
}

\begin{abstract}
Transmissions of multimedia data in emergency context such as, the occurrence of trauma or stroke are sensitive to the network delay, throughput and packet loss. Depending on the urgency, the communication channel quality can decide the survival of a patient. For saving lives, network infrastructure offering good Quality of Service (QoS), high broadband and low communication cost must be considered. In this context, Cognitive Radio Networks (CRN) seem appropriate. Indeed, this technology is well suited with its sensing ability and the scanning of a wide range of technologies, for assigning adequate frequency bands which meet the application constraints. In this paper, we propose a CRN-framework and an associated analytical tool that sets and imposes constraints to be respected, for ensuring reliable and high quality end-to-end communications.
\end{abstract}

\section{INTRODUCTION}

Public healthcare systems and tele-monitoring frameworks need innovation and more flexibility to meet the challenge of offering broadband networks. In fact, ehealth real-time applications and medical multimedia data transmissions require high quality of service and significant bandwidth for a better remote diagnosis. However, medical communication systems are increasingly constrained with limited resources and service cost. Indeed, communication architectures proposed for telemedicine applications are based on limited access technologies such as $3 \mathrm{G}$ or $4 \mathrm{G}$ not available everywhere. They also provide a quality of service, for example in terms of throughput which differs from one zone to another. Thus, deployment of efficient wireless networks infrastructure able to offer large frequency bands, will bring real opportunities for medical multimedia communication systems that could approach the standards of in-hospital examinations. The limitations and misuse of traditional Wireless Medical Telemetry Service (WMTS), Industrial Scientific and Medical bands (ISM), Ultra Wide Bandwidth (UWB), motivate and reinforce the idea of using Cognitive Radio Networks (CRN)[1] as a technology dedicated to emergency and health services. Cognitive Radio can greatly improve health services by its intrinsic characteristics, such as the use of licensed bands in addition to unlicensed bands usage, through the opportunistic access. In most common configurations, a Cognitive Radios (CR) user exploits the spectrum bands transparently to legacy users also called Primary Users (PU). Using its flexibility, the $\mathrm{CR}$ device explores different technologies and standards to identify free frequency bands for opportunistically ensuring its transmissions. The Cognitive Radio have recently become the forefront of wireless research, and promise to reduce connection cost and offer new applications as well as higher throughput to users. However, this technology requires additional reasoning tools for enhancing the performances under smart channel selection, reliable data communication with low delay and co-channel interference avoidance. In this paper, we propose a framework combined with the Model Predictive Control (MPC)[2] tool for Quality of Service assessment in Cognitive Radio Networks. The MPC-tool allows controlling the transmission parameters variation (throuput, transmission power, packet loss) and through its prediction ability, the MPC brings a dynamic adaptation of the input variables in order to meet the QoS requirements of the application. Our proposal therefore allows to guarantee transmissions quality in the context of multimedia eHealth applications that have high bandwidth requirements, end-to-end delay sensitivity and do not tolerate high packet loss rate. The remainder of this paper is structured as follows: in section II we make an overview on the related work and propose the framework for medical multimedia data transmission in section III. Then, we present the MPC-tool for the QoS control and prediction in section IV. In section V, simulation results are presented and analysed. Finally we conclude this work and point out some interesting perspectives in section VI.

\section{RELATED WORK}

Today, studies on delivering medical multimedia content focus on compression techniques, encoding methods, video resolution methods to meet the needs of adapting to the network individual parameters[3][4]. Adaptation to wireless parameters means in that case, diagnostically relevant selection, the source encoding parameters choice and error control methods for addressing transmission errors. E-health multimedia systems have been primarily based on $3 \mathrm{G}$ wireless networks[5]. However, given the limited upload data rates supported by these channels (up to $384 \mathrm{kps}$ ), as shown in [6], the associated source encoding parameters were bounded to different medical video resolution sizes/types. Researchers are now more focusing on $3.5 \mathrm{G}$ and $4 \mathrm{G}$ wireless technology for delivering high resolution and video rate with low delay and low packet-loss rate [7]. This evolution from $3 \mathrm{G}$ to $4 \mathrm{G}$ offers broadband multimedia transmissions but does not solve the spectrum scarcity issues and its inefficient usage. In this context, researchers are interested in the use of Cognitive Radio for medical multimedia transmissions. In [8], video encoding rate, power control, relay selection and channel allocation are jointly considered for video transmission over cooperative 
Cognitive Radio networks. The problem is formulated as a mixed-integer non-linear problem and solved by an algorithm based on a combination of the branch and bound framework and convex relaxation techniques. This contribution should take into account delay that is a very important constraint in real-time medical multimedia flow transmission. In [9], an algorithm is proposed for evaluating the expected delay of competing flows in single-hop and two-hop networks considering the time-varying spectrum condition and occupancy, traffic characteristics, and the condition of queues at intermediate nodes. Simulation results show that the proposed algorithm significantly reduces the packet loss rate and improves the average Peak Signal-to-Noise Ratio (PSNR) of the received video streams. The work presented in [10] considers the multiuser bit-rate and latency control of scalable video content in a Cognitive Radio multimedia network. The proposed model is based on CRN where multiple secondary users attempt to access a spectrum hole according to a predefined Time Division Multiple Access (TDMA) rule based on primary user activities, channel quality and transmission delay of each user. Scalable video rate and distortion models has been used in formulating the problem as a switching control dynamic Markovian game where video sources and channel behaviour have been modelled as independent Markov processes. This work shows that the proposed switching control game results in an improvement in video quality over a myopic rate allocation scheme in video PSNR. To the above mentioned transmission optimisation efforts, are added scheduling mechanisms used for traffic prioritization. In [11], a priority virtual queue model is adopted for wireless CR users to select channel and maximize video quality. Simulation results show that the proposed solution outperforms the conventional dynamic channel selection scheme by $2 \mathrm{~dB}$ (PSNR). Most of these research papers consider a single parameter to be improved, often the delay. The current state-of-the-art proposes also techniques for best channel selection or an algorithm for an efficient resource sharing in CRN. So, few studies have addressed the improvement of several parameters of quality of service at the same time. Also in practice, few researchers have focused on mechanisms to implement for allowing the Cognitive Radio nodes to have dynamic and automatic transmission parameters adaptation capability. In this paper, we propose an innovative idea that facilitates the optimization of the end-to-end transmission QoS by enabling the realtime control of transmission parameters and their dynamic adaptation through the use of MPC.

\section{THE PROPOSED MEDICAL MULTIMEDIA DATA-TRANSMISSION FRAMEWORK}

The success of this approach is undoubtedly related to sensing results, channel availability and initial route establishment as detailed in [12]. We assume that the sensing process is perfect and can provide a list of sufficient free channels to each Cognitive Radio node. Indeed, various research projects such as the $\mathrm{CREW}^{1}$ European project, experiment platforms for spectrum sensing tests in real environment. The provided results showed high accuracy in free bands detection. The results in [13][14] also showed the ability of a cognitive radio node to detect interference (such as primary user activity) in

\footnotetext{
${ }^{1}$ http://www.crew-project.eu/
}

real time and choose a communication strategy (frequency band change, transmission power adjustment), which is the most appropriate. With good quality of bandwidth, user should adopt the modulation-coding scheme that supports a higher data rate. Our proposal therefore, is based on existing channel modulation/encoding schemes, video compression types and video resolution/encoding specifications. Indeed, the proposed solution fits well and comes in complement of existing multimedia transmission functions as more described in [15]. As described in figure 1, medical imaging equipments such as ultrasounds, endoscopes, intra-oral cameras, surgical microscopes and sensors are devices that provide various medical data-1). Once medical multimedia data acquired, video resolution to match devices specification and data rate is considered. Multimedia resolution and frame rate adjustments-(2) aim to match the available bandwidth and end-user device capabilities. Diagnostically relevant[16] and encoding-(3) principle is to associate video regions of interest with clinical criteria. Medical multimedia contents are encoded using diagnosticallydriven and error resilient encoding. More precisely, to each sample of video is assigned a quality level based on its diagnostic significance namely, establishing the correspondence between the region of interest (image, video data) and the clinical significance. The Quantization Parameters (QP) associated

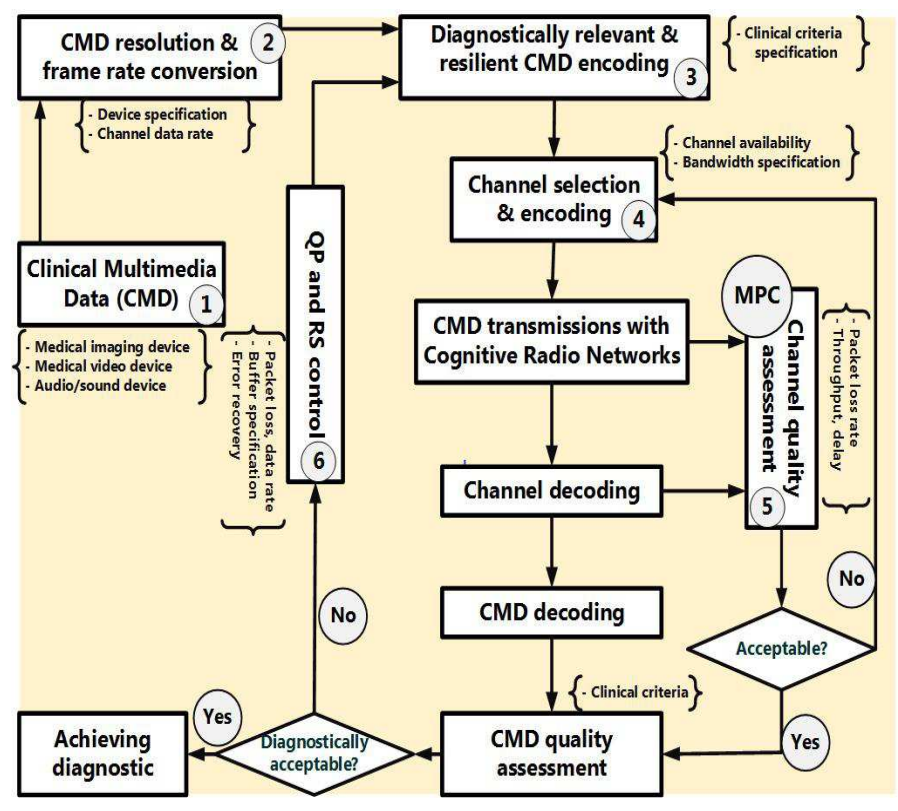

Fig. 1. Medical multimedia data transmission framework

to Reed-Solomon codes (RS)-(6) are important variables for quality of service consolidation and transmission parameters adjustment. Quantization Parameters are varying according to buffer, bandwidth and channel conditions. Reed-Solomon codes (RS) are used to correct transmission errors. Also, clinical video quality assessment methods [17] are essential for communicating reliable and good quality medical multimedia content to the medical experts [18]. Note that clinical specification and quantization parameters are associated to network parameters or the channel quality and channel selection criteria-(4). Thus, the proposed channel-control process allows frequency band selection according to the constraints of QoS. Transmission quality assessment-(5) with the MPC- 
module leads to estimate packet-loss rate, throughput and delay for useful adjustments. The criteria and conditions of transmissions are analysed using the proposed MPC-algorithm that allows to decide if frequency band change is needed or not. The automatic adaptation of network parameters and the constraints set by the MPC-module allow to mitigate delay and packets overdue that significantly affect the real-time transmission quality.

\section{CONTROLling QoS With The MPC}

Model Predictive Control (MPC) is a technique that adapts to the dynamics of a process by its real-time controller to predict the future behaviour of this process. Cognitive Radio is a communication technology based primarily on the time-slots principle. A time-slot is the period allocated by the scheduler or the resource sharing engine to a node for performing the operations of spectrum sensing, signalling process and data transfer on a given channel. Thus, when the quality of service degrades during a time-slot, the Cognitive Radio node can consider a change of frequency band for the next timeslot. The unstable nature of the transmission parameters and the Cognitive Radio channel should be controlled to optimize the use of resources for the future time-slots while taking into account the medical video quality. In our proposal, the MPC will make the optimal decision between staying on the current channel or move to other frequency band, according to the constraints and disturbances. These constraints are used to monitor the quality of service and become the basis for the decision-making. As instance, when the QoS is degraded, one of the possible decisions to make is to change the frequency band or the technology (WIFI, GSM). Therefore, the MPCtool will allow to follow the variation of the transmission parameters while ensuring their compliance with the established constraints. The main advantage of MPC compared to other statistical prediction models based on the history data, is the fact that it achieves optimized predictions by implementing only the current time slot with less calculation[19].

\section{A. Description of the MPC basic idea}

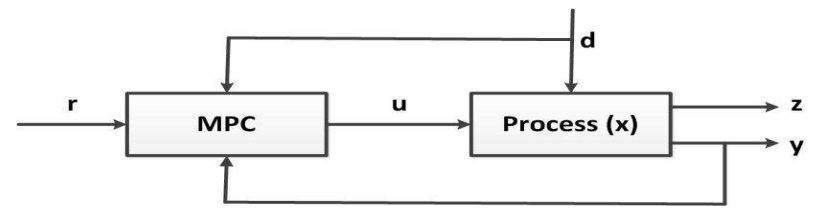

Fig. 2. The considered MPC-model

Figure 2 illustrates the basic idea for the MPC where $r$ is the set-points describing the reference values of transmission parameters. From these reference variables, the controller manipulates the inputs $u$, for achieving predictions through outputs $z$. The output values $z$ are then compared with measured values $y$, for ensuring the relevance of outputs while controlling their compliance with the set-points as possible. Variable $d$ is the disturbance linked to a primary user signal, and any other noise that could degrade transmissions quality. The principle is to monitor in real time the evolution (at time slot $t+1$ ) of the transmission parameters $(u)$ of medical data and make a decision on the basis of established criteria and constraints.

\section{B. Modelling the MPC for QoS monitoring}

The MPC is based on a transfer function $\mathrm{H}(\mathrm{x})$ that expresses the relation between each input $u$ and output $z$ variables as follow: $H(x)=\frac{Z(x)}{U(x)}$.

We then deduce from $\mathrm{H}(\mathrm{x})$, the following optimization function given below:

$\phi=\frac{1}{2} \sum_{i=1}^{N} w_{z_{i}}\left\|\left(z_{i}-r_{i}\right)\right\|^{2}+\frac{1}{2} \sum_{i=1}^{N-1} w_{u_{i}}\left\|\Delta u_{i}\right\|^{2}$

And with $\phi$, we can predict the future sequence, when there exist $n$ transmission parameters to control (output variables) and $\mathrm{m}$ other transmission parameters to manipulate as inputs. This prediction is detailed by the function:

$$
\begin{aligned}
\Phi= & \sum_{i=1}^{n}\left(\sum_{j=1}^{j=N_{z_{i}}} w_{z_{i}}\left\|\left(z_{i}(t+j)-r_{i}(t+j)\right)\right\|^{2}\right)+ \\
& \sum_{i=1}^{m}\left(\sum_{j=1}^{j=N_{u_{i}}} w_{u_{i}} \|\left(z_{i}\left(\Delta u_{i}(t+j-1)\right) \|^{2}\right)\right.
\end{aligned}
$$

Coefficients $w_{z_{i}}$ and $w_{u_{i}}$ are weights that can give more importance to a given parameter. To take into account the constraints of the transmission environment, MPC offers the possibility to assign different types of constraints (output and input) on the network parameters. These constraints can be summarized as follow:

Prediction $x_{i+1}=A x_{i}+B u_{i}+E d_{i}$ for $i=0,1,2, \ldots, N-1$ Where:

$$
\begin{gathered}
z_{i}=C x_{i}, \text { for } i=0,1,2, \ldots, N \\
u_{\min } \leq u_{i} \leq u_{\max }, \text { for } i=0,1,2, \ldots, N-1 \\
\Delta u_{\min } \leq \Delta u_{i} \leq \Delta u_{\max }, \text { for } i=0,1,2, \ldots, N-1 \\
z_{\min } \leq z_{i} \leq z_{\max }, \text { for } i=0,1,2, \ldots, N
\end{gathered}
$$

We can note that the prediction at time-slot $t_{0}$ is not considered, and that gives the future values with the following constraints:

$$
\begin{gathered}
z_{\min } \leq z_{i} \leq z_{\max } \Longrightarrow \\
{\left[\begin{array}{c}
z_{\min } \\
z_{\min } \\
\cdot \\
\cdot \\
z_{\min }
\end{array}\right] \leq\left[\begin{array}{c}
z_{1} \\
z_{2} \\
\cdot \\
\cdot \\
z_{N}
\end{array}\right] \leq\left[\begin{array}{c}
z_{\max } \\
z_{\max } \\
\cdot \\
\cdot \\
z_{\max }
\end{array}\right]}
\end{gathered}
$$

A Cognitive Radio node could continue to occupy a given channel-band for its transmission of medical data only if these constraints are respected. The fixed constraints help ensure and guarantee a good quality of service for multimedia medical data transmission.

\section{Basis of QoS criteria selection in this context}

The project DATAR ${ }^{2}$ initiated in France, allows to develop a guide which provides important recommendations for good

\footnotetext{
${ }^{2}$ www.datar.gouv.fr
} 


\begin{tabular}{|l||c|c|c|}
\hline \multicolumn{1}{|c|}{ Bandwidth } & $2 \mathrm{MB}$ & $4 \mathrm{MB}$ & $8 \mathrm{MB}$ \\
\hline \hline Image-type & $49 \mathrm{sec}$ & $24,5 \mathrm{sec}$ & $12 \mathrm{sec}$ \\
\hline Abdominal scan(600 images) & $8 \mathrm{~min}$ & $4 \mathrm{~min}$ & $2 \mathrm{~min}$ \\
\hline TAP scan(1000 images) & $13,20 \mathrm{~min}$ & $6,40 \mathrm{~min}$ & $3,20 \mathrm{~min}$ \\
\hline Radio *4 incidences & $37 \mathrm{sec}$ & $18 \mathrm{sec}$ & $9 \mathrm{sec}$ \\
\hline
\end{tabular}

TABLE I. EXAMPLE OF TRANSMISSION DELAY VARIATION BETWEEN A HEALTH CENTER AND A SERVER

\begin{tabular}{|l||c|c|c|c|}
\hline Bandwidth & $1 \mathrm{MB}$ & $2 \mathrm{MB}$ & $6 \mathrm{MB}$ & $8 \mathrm{MB}$ \\
\hline \hline Brain scan & $31 \mathrm{sec}$ & $15 \mathrm{sec}$ & $5 \mathrm{sec}$ & $4 \mathrm{sec}$ \\
\hline Abdominal scan & $5,07 \mathrm{~min}$ & $2,34 \mathrm{~min}$ & $51 \mathrm{sec}$ & $38 \mathrm{sec}$ \\
\hline TAP scan & $8,32 \mathrm{~min}$ & $4,16 \mathrm{~min}$ & $1,25 \mathrm{~min}$ & $1,04 \mathrm{~min}$ \\
\hline Radio & $32 \mathrm{sec}$ & $16 \mathrm{sec}$ & $5 \mathrm{sec}$ & $4 \mathrm{sec}$ \\
\hline
\end{tabular}

TABLE II. EXAMPLE OF TRANSMISSION DELAY VARIATION BETWEEN A SERVER AND A MOBILE RADIOLOGY CENTER

practice in telemedicine infrastructure deployment. Their work consisted, to gather experiences in 8 pilot regions and then compare the needs and barriers identified, depending on operators. Analyzes and experiences in this project show that the most significant variable for QoS remain the stability of the connection, its availability and the performance in term of throughput, latency, jitter and packet loss. Thus, on the basis of experiments, measurements, the analysis of the transfer time depending on the bandwidth and data type has been provided in Table I and II. A set of suggestions have been also made for determining the limits to be considered in e-health network infrastructures. Table I is an example of transfer time between a healthcare facility and a central server. Table II is an example of transfer time between the central server and a mobile radiology center. These two examples provide values for the analysis of transmission delay in different context (mobile or static) and obviously different distances. These two tables, inspired by the values in DATAR guide show that the transmission rate depends on the selected bandwidth and also is linked to the type of image to be transmitted for a given distance. Thus the decision-making module of the Cognitive Radio will consider these context information to select the frequency band and the technology to be used. Based on this information, the urgency of the transmission and the priority level of the medical stream, the constraints are set to provide the best service. An overview of classes of service and their related priority level used in the DATAR guide is given in Table III. Table III is an example of flow classification which served as a reference for recommendations in term of acceptable delay, packet loss and throughput. Thus, the acceptable latency for real-time class flow is estimated between 10 to 20 milliseconds for a datagram of 100 bytes, according to geographical area and the type of channel used. This latency is considered suitable when it is between 20 to

\begin{tabular}{|l||c|c|}
\hline Priority class & Weight & Services \\
\hline \hline 1 & ++++ & Real-time traffic (voice over IP) \\
\hline 2 & +++ & telediagnosis, telemedecine traffic \\
\hline 3 & ++ & Monitoring traffic \\
\hline 4 & $=$ & best effort traffic (web, mail) \\
\hline 5 & - & non-priority traffic (backup, FTP) \\
\hline \multicolumn{2}{|c|}{ TABLE III. } & EXAMPLES OF SERVICE CLASS
\end{tabular}

40 milliseconds for the other stream classes and for the same amount of data. The packet loss rate is also fixed to $0.2 \%$ and the jitter to 5 milliseconds by this experiment results for a datagram of 100 bytes. The results of these experiments are the basis for our MPC model-simulation parameters selection.

\section{Implementation of the QoS-control with MPC}

Controlling the quality of service in Cognitive Radio Networks through the MPC tool will be based on the following principle:

- Evaluation of transmission parameters: throughput, packet loss, delay, which are values that can be obtained through real-time QoS measurement/statistic tools such as the Quality of Service Metrology (QoS$\mathrm{Met}^{3}$ ) and the channel availability information is given by the sensing module. It should be noted that the availability of the channel should be at least $99.99 \%$ of the time of transmission to ensure proper connectivity.

- Decision: At each time slot $t$, the MPC module calculates and predicts transmission conditions (variation of parameters) of time slot $t+1$.

1) If transmission conditions are good for the time slot $t+1$ then, no channel change mechanism should be considered. This means that for the time slot $t+1$, there is a high probability of maintaining the quality of transmission to an acceptable level for the medical multimedia data.

2) Otherwise, a beacon message is sent through the signalling channel for a channel reservation as detailed in [20][21] or for requesting a new channel in time slot $t+1$. This reflects a requirement to change frequency band to meet the constraints of quality of service.

We used Matlab for implementation of the QoS monitoring in medical multimedia data transmission context. These simulations are performed according to the relevance of parameters, in real-time multimedia data transfer context. These transmission variables are: delay, packet size, throughput, bandwidth, packet loss, transmit power and channel threshold. For each parameter, we defined the inherent constraints as detailed in Table IV. The limits in Table I, are all constraints to be respected by the Cognitive Radio node to continue using the channel-band without triggering channel hopping process. The frequency hopping or the technology change process is triggered only when these instructions and these constraints are violated. This guarantees a better use of the available freebands and offers an efficient sharing of the spectrum resources. The Matlab code in Figure 3 shows how this control of variable is implemented in practice. The parameters chosen for the purpose of these simulations are based on values shown in the accompanying guide (DATAR).

Thus, the recommended delay for real-time telemedicine applications has to be less than 5 milliseconds for 100 bytes of data. The minimum throughput to ensure quality of service is $10 \mathrm{Mbps}$ and packet loss is fixed to $0.2 \%$ maximum for 100 bytes of data. Knowing also that the multimedia transmission

\footnotetext{
${ }^{3} \mathrm{http}: / /$ michaut.valerie.free.fr/qosmet/
} 


\begin{tabular}{|l|c|}
\hline Parameters & Descriptions \\
\hline \hline $\mathrm{N}$ & Prediction horizon size \\
\hline$u_{\min }$ & Lower limit of each transmission parameter linked to $u$ \\
\hline$u_{\max }$ & Upper limit of each transmission parameter linked to $u$ \\
\hline$\Delta u_{\min }$ & Rate of change of the parameter $u_{\min }$ \\
\hline$\Delta u_{\max }$ & Rate of change of the parameter $u_{\max }$ \\
\hline$z_{\min }$ & Lower limit of each predicted parameter \\
\hline$z_{\max }$ & Upper limit of each predicted parameter \\
\hline$x_{0}, u_{0}$ & Simulation starting points \\
\hline $\mathrm{w}$ & Weight matrices related to each parameter importance \\
\hline & TABLE IV. PARAMETERS DEFINITION
\end{tabular}

needs a large bandwidth then we set the following constraints that could be dynamic, adaptable and change depending on the technology and the radio environment. The important details

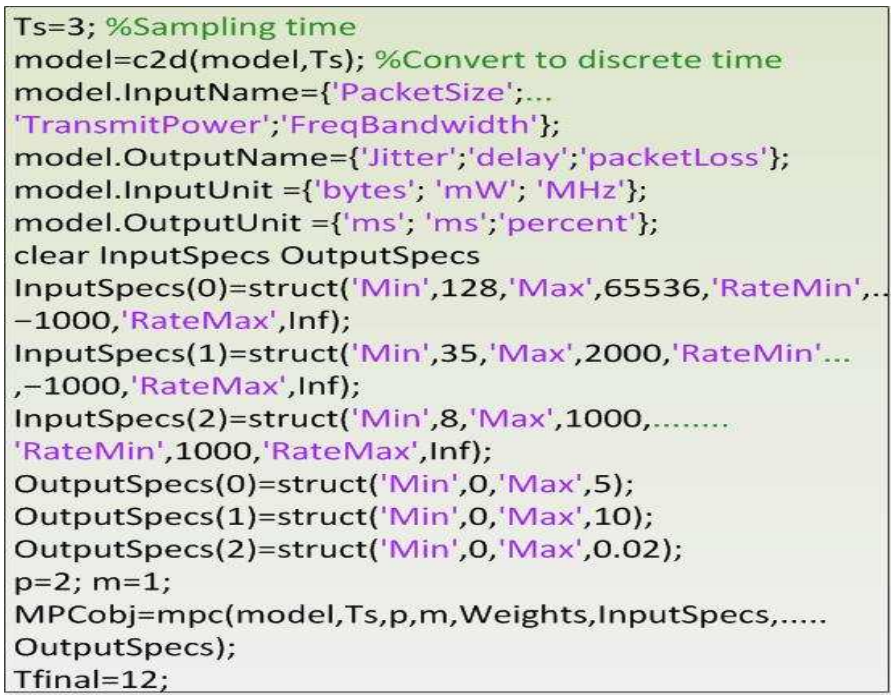

Fig. 3. Constraints set for simulation in Matlab

in this code are relating to the limits fixed to the variables (InputSpecs,OutputSpecs), that are the constraints to be observed. For simulations, we set a couple of value (RateMin, RateMax) that determines the frequency of changes of each variable and another value $(p)$ for the prediction horizon. Variables jitter, delay and packet loss are calculated based on a datagram of 100 bytes. Packet size parameter is determined based on the characteristics of the underlying technology.

\section{RESUlTS AND ANALYSIS}

Before describing the results, it should be noted that the MPC toolbox functions (autosc, scal and wrtreg) allows to arrange all data (input variables and output variables) in the same order of magnitude. The predictions and results obtained by simulation are based on these re-estimated values. The objective remains minimizing a quadratic criterion based on prediction errors up to an order equal to the relative degree between the predicted outputs/inputs variables and the reference values often measured. The different results thus show the vectors of predicted future errors over the horizon $p$ which result from all future manipulated variables. The main objective is to reach a stability of the system in time-varying manipulate variables (inputs, outputs). This stability reflects

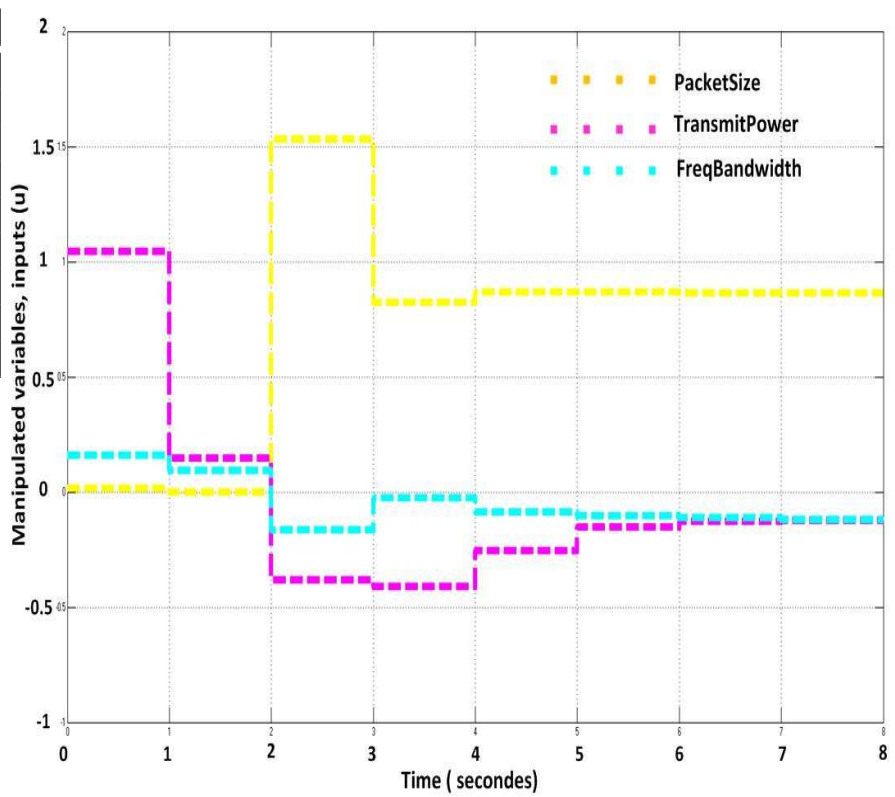

Fig. 4. Input variables variation

the fact that the desired QoS, with constraints on the output variables is achieved through dynamic adaptation of the input parameters (packet size, transmit power, frequency bandwidth). Indeed, we set the constraints/limits on input and output variables and the input parameters are dynamically adapted as described at Figure 4. These simulations also take into account the prediction horizon $(p)$ to assess the degree of accuracy of the predicted values. The variable $p$ can help make a choice on the frequency of calculation/execution of the algorithm for the predictions in future time-slots. For efficiency reason, it was interesting to know whether one prediction would be sufficient for a number of time-slots. The obtained results show that the accuracy of the predictions depends on the prediction horizon variable. More the prediction horizon is far with $p=5$, less the results are accurate. Even if $p$ variable with greater value has the advantage of providing a low computation/execution rate, for the QoS control which remains the main objective of our contribution, the prediction horizon set at a close value $(p=$ 2) seems quite sufficient. However, It could be interesting to find a compromise between cost/complexity of calculation and the accuracy of predictions through the choice of prediction horizon value.

As regards the control of the QoS through the MPCfunction, the results of the Figure 5 show the case where the set constraints are not met. This means that the conditions to ensure an acceptable level of QoS are not met on the given frequency band. In case the system fails to stabilize (Figure 5) or it loses its stability during a time-slot, then frequency hopping for next timeslot becomes relevant. Figure 6 with a prediction horizon $p=5$ confirms the inaccuracies in the predicted values and also allows to see that the system hardly stabilizes by reaching the equilibrium only from $t=$ 6. For a close value prediction horizon such as $\mathrm{p}=2$, the equilibrium state has been faster reached and predictions are more accurate as shown in Figure 7. With this result (Figure 7) which reflects the best formulation for QoS-control through the MPC, the most important behaviour to be analysed and to 


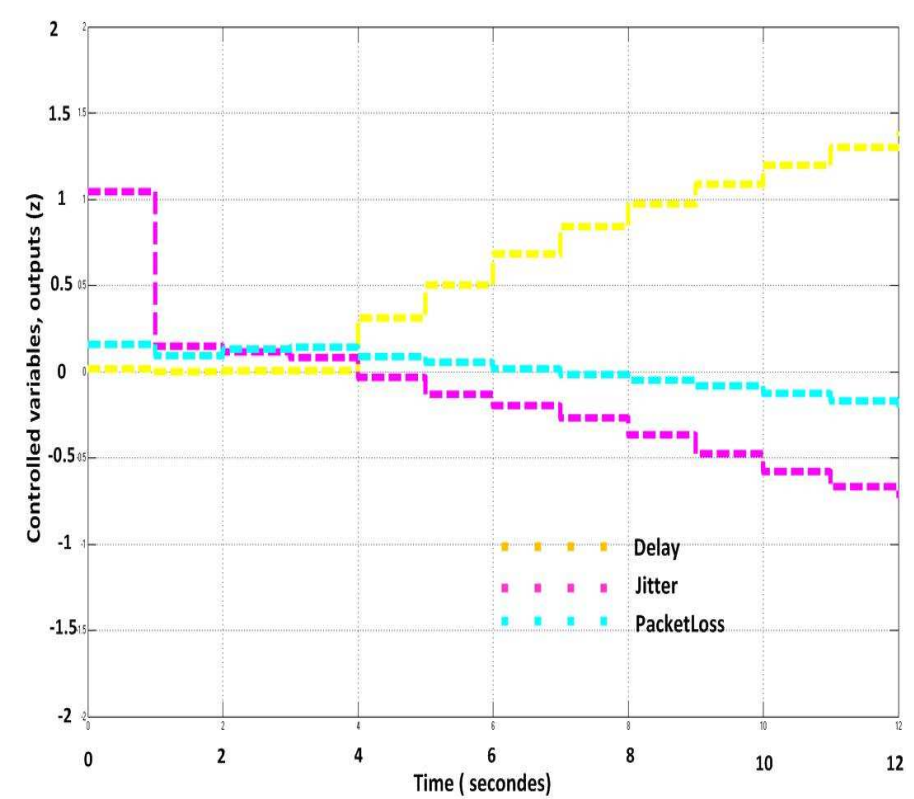

Fig. 5. Output variables variation without equilibrium

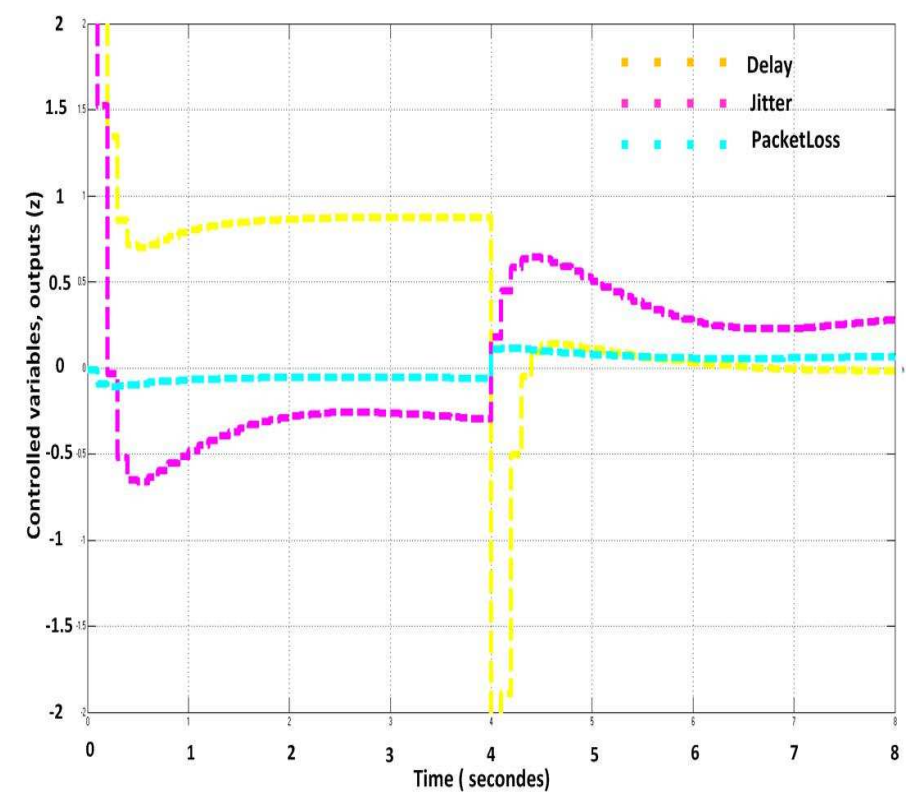

Fig. 6. Output variables variation with equilibrium for $\mathrm{p}=5$

note is the period when the system gets equilibrium. As long as this equilibrium remains, it means that the quality of service expressed through the constraints (delay, jitter, packet loss) is observed. The great advantage from these results is to have shown that in our system with Cognitive Radio equipments, it becomes possible to fix constraints on the output and input variables, and the Cognitive Radio equipment makes dynamic choice of parameter values in the range of the fixed intervals. This allows the equipments to keep their adaptable character by choosing their transmission parameter values based on the radio environment and the underlying constraints. In summary, one of the characteristics of this framework is that it opens up new perspectives in improving the quality of service for the transmission of medical data which are very sensitive to

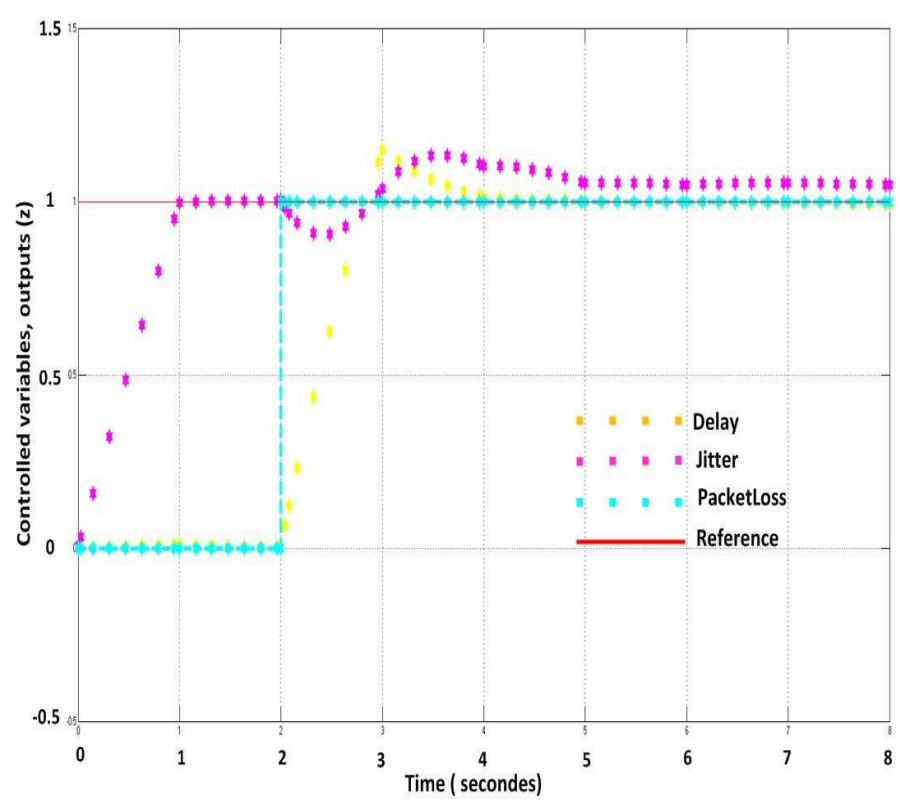

Fig. 7. Output variables variation with equilibrium for $\mathrm{p}=2$

delay and packet loss. In fact, most of the work particularly focused on compression algorithms as we have described in the related work for improving the QoS. About our proposal, we focused on the choice of the network infrastructure, on the dynamic adaptation of transmission parameters and on the more adequate channel selection/reservation for improving the overall QoS. Thus, this framework of control parameter and searching/selection of appropriate frequency band that we propose could be a real alternative to image compression techniques which inevitably deteriorate the quality of multimedia content provided to clinicians. Indeed, the compression ratio could be limited if the network infrastructure is able to provide the resources (bandwidth) required to guarantee the QoS.

\section{CONCLUSION}

In this paper, we proposed the Cognitive Radio as network infrastructure suitable for the transmission of medical multimedia data. We have proposed a framework that allows the transmission of multimedia medical data in Cognitive Radio networks with high demands of quality of service. Thus, for improving performance and provide better quality of service, we integrate in this framework a quality of service control function based on MPC-tool. This function controls the transmission parameters and allows decision-making (channel or technology change, transmit power and packet size adaptation) when the required QoS is no longer available. More precisely, our proposal allows to highlight the following main contributions. Firstly, transmission of medical multimedia content that we propose, is based on a distributed decision making scenario. The neighbouring nodes (or relay nodes) exchange information, about the priority level, the content type through the signalling channel. Based on the received information, each Cognitive Radio node decides on the value of its transmission parameters, depending on available resources and QoS constraints. Secondly, with the control of input/output parameters and dynamic adjustment of input variables offers by the MPC-tool, it becomes possible at each intermediate 
node to negotiate the best variable values to ensure the QoS. The assignment of a frequency bandwidth becomes dynamic and varies according to the urgency of medical multimedia content to be transmitted. Finally, the user is no longer forced to leave the channel with the detection of a primary user. It becomes possible to adapt the transmission parameters (e.g. transmission power) and continue sharing the channel. The future work will allow us to implement this solution on our Cognitive Radio platform LICoRNe, to evaluate its experimental performances, computational cost and energy consumption. We will also compute and evaluate the value of the prediction horizon $(p)$ which offers the best compromise between complexity/cost calculation and accuracy of predicted values.

\section{ACKNOWLEDGMENT}

This work is supported by the LICoRNe project, funded in part by the National Agency for Research in France ANR (Agence Nationale de la Recherche).

\section{REFERENCES}

[1] Mitola, J.; Maguire, G.Q., Jr. , Cognitive radio: making software radios more personal, Personal Communications IEEE , vol.6, no.4, pp.13,18, Aug 1999.

[2] Zhang Ju; Wan Senlin, A review of explicit model predictive control, Control Conference (CCC), 2012 31st Chinese, July 2012.

[3] Jingfang Huang; Zhaoyang Zhang, Honggang Wang; Hong Liu, Video transmission over Cognitive Radio networks, GLOBECOM Workshops (GC Wkshps), 2011 IEEE, 2011.

[4] Mingjing Ai; Dairui Cui; Shaopeng Tang; Ya Zhang, Network Adaptable Transmission Strategy Applied to H.264, Digital Media and its Application in Museum and Heritages, Second Workshop, pp.312,317, Dec. 2007.

[5] Panayides, A.; Pattichis, M.S.; Pattichis, C.S.; Schizas, C.N.; Spanias, A.; Kyriacou, E., An overview of recent end-to-end wireless medical video telemedicine systems using 3G, EMBC Annual International Conference, IEEE, 2010.

[6] Panayides, A.; Pattichis, M.S.; Pattichis, C.S.; Loizou, C.C.; Pantziaris, M.; Pitsillides, A., Atherosclerotic Plaque Ultrasound Video Encoding, Wireless Transmission, and Quality Assessment Using H.264 :, IEEE Transactions, 2011.

[7] Peter Rysavy, Transition to 4G : 3GPP Broadband Evolution to IMTAdvanced (4G), 3G Americas Publishes Research Report on 3GPP Mobile Broadband Evolution, September 2010.

[8] Zhangyu Guan; Lei Ding; Melodia, T.; Dongfeng Yuan, On the Effect of Cooperative Relaying on the Performance of Video Streaming Applications in Cognitive Radio Networks, Communications (ICC), IEEE International Conference, June 2011.

[9] Lei Ding, Scott Pudlewski, Tommaso Melodia, Stella N. Batalama, John D. Matyjas, Michael J. Medley, Distributed Spectrum Sharing for Video Streaming in Cognitive Radio Ad Hoc Networks, ADHOCNETS, Canada 2009.

[10] Mansour, H.; Huang, J.W.; Krishnamurthy, V., Multi-user scalable video transmission control in cognitive radio networks as a Markovian dynamic game, Proceedings of the 48th IEEE Conference 2009.

[11] Hsien-Po Shiang; van der Schaar, M., Dynamic channel selection for multi-user video streaming over cognitive radio networks, Image Processing, ICIP, 15th IEEE Inter. Conference, 2008.

[12] Dramane Ouattara, Francine Krief, Mohamed A. Chalouf, Omessaad Hamdi, Spectrum Sensing Improvement in Cognitive Radio Networks for Real-Time Patients Monitoring, Wireless Mobile Communication and Healthcare, Springer 2013, p179-188.

[13] Viswanathan, R., Cooperative spectrum sensing for primary user detection in cognitive radio, Sensing Technology (ICST), 2011 Fifth International Conference 2011.
[14] Ouattara, D.; Quach, M.T.; Krief, F.; Chalouf, M.A.; Khalife, H., Mitigating the hospital area communication's interference using Cognitive Radio Networks, e-Health Networking, Applications and Services (Healthcom), 2013 IEEE 15th International Conference, pp.324,328, 912 Oct. 2013.

[15] Andreas S. Panayides and Anthony G. Constantinides, DRIVEN: Diagnostically Robust Ultrasound Video Transmission over Emerging Wireless Networks, in ERCIM News 95, Special theme: Image Understanding, October 2013 (56 pages).

[16] Panayides, A.; Antoniou, Z.C.; Mylonas, Y.; Pattichis, M.S.; Pitsillides, A.; Pattichis, C.S., High-Resolution, Low-Delay, and Error-Resilient Medical Ultrasound Video Communication Using H.264/AVC Over Mobile WiMAX Networks, Biomedical and Health Informatics, IEEE Journal 2013.

[17] Lopez, J.P.; Jimenez, D.; Cerezo, A.; Menendez, J.M., Noreference algorithms for video quality assessment based on artifact evaluation in MPEG-2 and H.264 encoding standards, IFIP/IEEE International Symposium, pp.1336,1339, May 2013.

[18] Cavero, E.; Alesanco, A.; Garcia, J., Enhanced Protocol for Real-Time Transmission of Echocardiograms Over Wireless Channels, Biomedical Engineering, IEEE Transactions 2012.

[19] Jian-suo zhou; Zhi-Yuan Liu; Run Pei, A new nonlinear model predictive control scheme for discrete-time system based on sliding mode control, American Control Conference, 2001.

[20] Jin Lai; Ren Ping Liu; Dutkiewicz, E.; Vesilo, R., Optimal Channel Reservation in Cooperative Cognitive Radio Networks, Vehicular Technology Conference (VTC Spring), IEEE 73rd, 2011.

[21] Shu-Hsien Wang; Chih-Yu Hsu; Hong, Y.-W.P., Distributed Exploitation of Spectrum and Channel State Information for Channel Reservation and Selection in Interweave Cognitive Radio Networks, Wireless Communications, IEEE Transactions 2013. 